\title{
modélisation du comportement d'une craie blanche très poreuse et validation
}

\author{
modelisation of behaviour \\ of a white high porous chalk and validation
}

\author{
J.F. SHAO, J.P. HENRY \\ Laboratoire de Mécanique de Lille \\ Département de Mécanique des Matériaux Fragiles*
}

\begin{abstract}
Résumé
Des essais de compression isotrope ef triaxiale de révolution jusqu'à des pressions de confinement de $50 \mathrm{MPa}$ ont été effectués sur une craie blanche très poreuse. Les résultats obtenus ont permis de mettre en évidence les aspects fondamentaux de son comportement.

Le modèle élasto-plastique à deux surfaces de charge de LADE a été utilisé pour la modélisation: certaines modifications importantes ont été proposées pour prendre en compte les particularités du comportement de la craie.

Un premier test de validation du modèle proposé a été effectué dans des problèmes homogènes : chemins de sollicitations proportionnelles et d'extension latérale. De façon générale, de très bonnes concordances entre les résultats expérimentaux et la simulation par le modèle ont été obtenues.

Un deuxième test de validation est présenté pour des champs de contraintes non uniformes: essais sur cylindres creux. Un programme de calcul en éléments finis, dans lequel le modèle de LADE modifié est introduit, a été mis au point et a servi à simuler ces essais. La comparaison entre l'expérience et la simulation a également été faite et permet d'apprécier la bonne aptitude du modèle à traduire le comportement de la craie.
\end{abstract}

\footnotetext{
Abstract

Hydrostatic compression and conventional triaxial compression tests were performed on a white, highly porous chalk for confining pressure up to $50 \mathrm{MPa}$. The obtained results have allowed to show up fundamental aspects of the chalk behaviour. A LADE elastoplastic model with two yield surfaces was used for the modelisation: some important modifications were proposed for taking into account particularities of the chalk behaviour. Proportional loading test and triaxial lateral extension test were performed tc study chalk's behaviour in homogeneous problems. The modified LADE elastoplastic model was used to simulate these tests. In global way, model's predictions were found to be in satisfactory agreement with the experimental results.

Finally, hollow cylinder tests were realized to examine chalk's behaviour in typical boundary conditions problems. The modified elastoplastic model has then been introduced into a finite elements program which was used to simulate the hollow cylinder tests. Comparisons between experimental and predicted results were also given.
} 


\section{INTRODUCTION}

Pour le calcul et l'étude de stabilité des ouvrages dans les milieux rocheux (tunnels, galeries, cavités et forages pétroliers profonds), la connaissance d'un modèle rhéologique interprétant correctement le comportement des roches est fondamentale et nécessaire.

L'objectif visé dans notre étude est de modéliser le comportement rhéologique d'une craie blanche sous les sollicitations statiques et monotones jusqu'à des pressions hydrostatiques de $50 \mathrm{MPa}$. L'intérêt de cette modélisation se situe essentiellement dans l'étude de la stabilité des forages pétroliers profonds et des subsidences induites [8]. MILLER et CHEATHAM [7], ont fait l'un des premiers travaux sur la modélisation du comportement des roches calcaires poreuses. Ils ont proposé un modèle élastoplastique à deux surfaces de charge en utilisant le critère de COULOMB pour la surface de rupture et deux plans pour la cap-surface de charge. A cette modélisation, on peut reprocher que le critère de COULOMB n'est pas bien vérifié par la plupart des roches poreuses, et que la cap-surface de ce modèle possède un régime d'arête qui entraîne des difficultés numériques de résolution [10]. Plus récemment, ELLIOTT et BROWN [2], [3] ont réalisé une étude expérimentale sur des roches calcaires de porosité moyenne, et ont étudié le critère d'écoulement plastique à partir du concept d'état critique appliqué aux roches par GEROGIANNOPOULOS [4], mais il a été constaté que le concept d'état critique ne présente plus de signification pour des roches poreuses sous sollicitations à pression hydrostatique importante. CHEATHAM et al. se sont intéressés à l'anisotropie introduite par l'histoire de sollicitations en travaillant sur des lois d'écrouissage cinématique [1].

Cette roche présente un comportement bien particulier à cause de la forte porosité : sous une pression hydrostatique faible, elle se comporte comme une roche fragile; et sous une pression hydrostatique élevée, comme un sol. Entre ces deux zones, il y a destruction progressive de la microstructure rendant incohérents les grains de calcite constituant la roche. La difficulté majeure pour modéliser le comportement d'une telle roche est d'interpréter la transition d'un comportement à l'autre par simple évolution des paramètres du modèle retenu, et de traduire la contractance plastique due aux cisaillements.

\section{ASPECTS FONDAMENTAUX DU COMPORTEMENT DE LA CRAIE}

\subsection{Caractérisation de la craie et conditions d'essais}

Les essais de compression isotrope et triaxiale ont été effectués pour étudier les aspects fondamentaux du comportement de la craie.

La craie étudiée est une craie blanche typique, provenant de la carrière d'Haubourdin au nord de la France. Cette craie de lithologie sénonienne est caractérisée par une forte teneur en $\mathrm{CaCO}_{3}(96,60 \%)$ et une porosité très élevée (45\%).

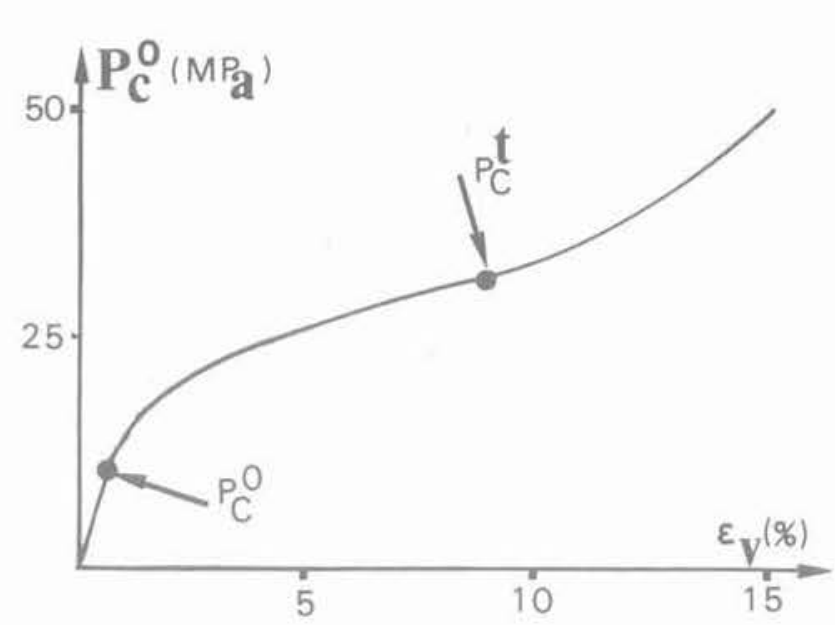

Fig. 1. - Essai de compression isotrope.

Fig. 1. - Hydrostatic compression test.

Les éprouvettes cylindriques ont été soigneusement préparées à partir des carottes prélevées in-situ. Elles ont été séchées à une température de $100^{\circ} \mathrm{C}$ pendant 24 heures, puis saturées sous vide ou sous pression interstitielle pendant 12 heures avec du méthanol, considéré comme neutre chimiquement vis-à-vis du comportement mécanique de la craie. Nous avons utilisé des éprouvettes de grande dimension dont les cotes géométriques moyennes sont les suivantes: diamètre $110 \mathrm{~mm}$, hauteur $124 \mathrm{~mm}$.

Le dispositif expérimental utilisé est composé essentiellement d'une presse hydraulique asservie et d'une cellule haute pression de grande dimension [10]. Ayant utilisé des échantillons saturés, nous avons déterminé la variation de volume à partir du volume de méthanol entrant ou sortant dans les éprouvettes. Ceci a été basé sur l'hypothèse que la déformabilité du squelette de la craie est négligeable devant la variation de volume des vides. Cette hypothèse a été vérifiée en comparant la mesure donnée par le capillaire (variation de volume des vides), et celle par les jauges d'extensométrie (variation de volume global) pour un essai de compression isotrope jusqu'à $16 \mathrm{MPa}[10]$.

Les essais triaxiaux ont été réalisés en force contrôlée. Afin de respecter la condition de sollicitation quasi statique, et d'avoir des courbes contraintes-déformations les plus proches possible de la réalité, c'est-à-dire par rapport aux conditions d'exploitation des résultats du présent travail, la vitesse de sollicitation utilisée a été choisie très faible ( $\cong 10^{-3} \mathrm{MPa} / \mathrm{s}$ ).

\subsection{Comportement de la craie dans des essais de compression isotrope}

La figure 1 représente une courbe typique de variation de volume globale $\varepsilon_{v}$ en fonction de la pression hydrostatique $\mathrm{P}_{c}$, obtenue pour la craie étudiée dans des essais de compression isotrope jusqu'à $50 \mathrm{MPa}$. A partir de cette courbe expérimentale, nous pouvons faire les remarques suivantes : 
1. Cette courbe présente trois phases distinctes : lorsque la pression hydrostatique est supérieure à $\mathrm{P}_{\mathrm{c}}^{\circ}$, nous avons une phase de forte contractance plastique jusqu'à une certaine valeur $\mathrm{P}_{c}^{t}$. Cette phase correspond à un effondrement progressif de la structure microporeuse de la craie, par rupture des ponts entre grains. $\mathrm{Si}$ la pression hydrostatique est supérieure à $\mathrm{P}_{c}^{1}$, il s'agira d'une phase d'écrouissage croissant avec diminution rapide du taux de contraction plastique, qui est une conséquence de la croissance de surface de contact entre les grains.

2. L'effondrement progressif plastique de la microstructure fait diminuer la porosité de la craie. Des essais au porosimètre à mercure ont montré que la pression hydrostatique a détruit essentiellement des pores de rayon supérieur à $3000 \AA$.

3. En effectuant un certain nombre de cycles de déchargement rechargement, nous avons déterminé le module de compressibilité isotrope à chaque point de déchargement, c'est-à-dire pour différentes valeurs de la pression hydrostatique ; les valeurs de ces modules ainsi déterminées sont reportées sur la figure 2. Nous avons constaté que ce module de compressibilité isotrope $\mathrm{K}_{\mathrm{ur}}$ augmente en fonction de la pression hydrostatique.

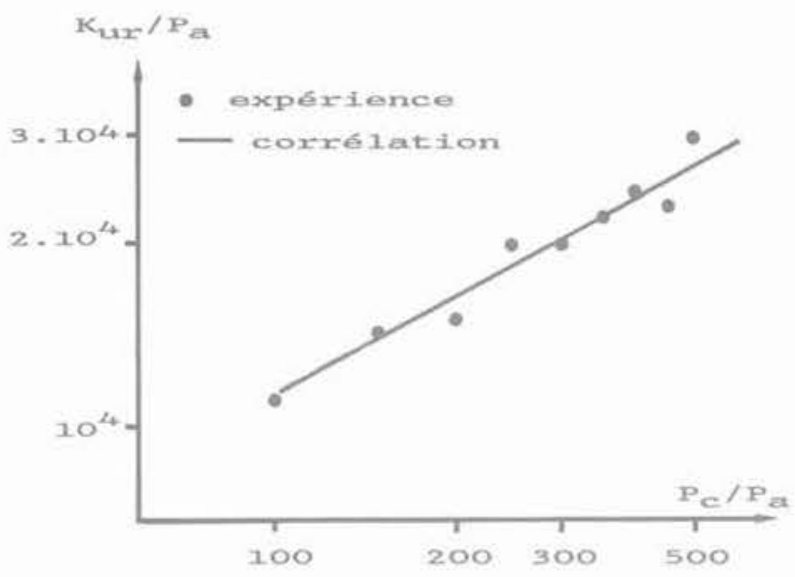

Fig. 2. - Variation du module de compressibilité et corrélation.

Fig. 2. - Variation of bulk modulus and correlation.

\subsection{Comportement de la craie dans des essais de compression triaxiale}

Une série d'essais triaxiaux de révolution en compression ont été effectués sur la craie blanche. Les pressions de confinement utilisées sont les suivantes : 2,4 , $5,7,10,15,20,30,40$ et $50 \mathrm{MPa}$. La figure 3 présente les courbes contraintes-déformations longitudinales et les courbes contraintes-déformations volumiques pour trois pressions de confinement représentatives : une faible $\left(\sigma_{3}=4 \mathrm{MPa}<\mathrm{P}_{\mathrm{c}}^{\circ}\right)$, une moyenne $\left(\mathrm{P}_{\mathrm{c}}<\sigma_{3}\right.$ $\left.=20 \mathrm{MPa}<\mathrm{P}_{c}^{\prime}\right)$ et une élevée $\left(\sigma_{3}=40 \mathrm{MPa}>\mathrm{P}_{\mathrm{c}}^{\prime}\right)$. A partir de ces résultats expérimentaux, nous pouvons faire les constatations suivantes :

1. Pour les essais à pression de confinement inférieure à $\mathrm{P}_{c}^{\circ}$, nous observons des pics de résistance sur la

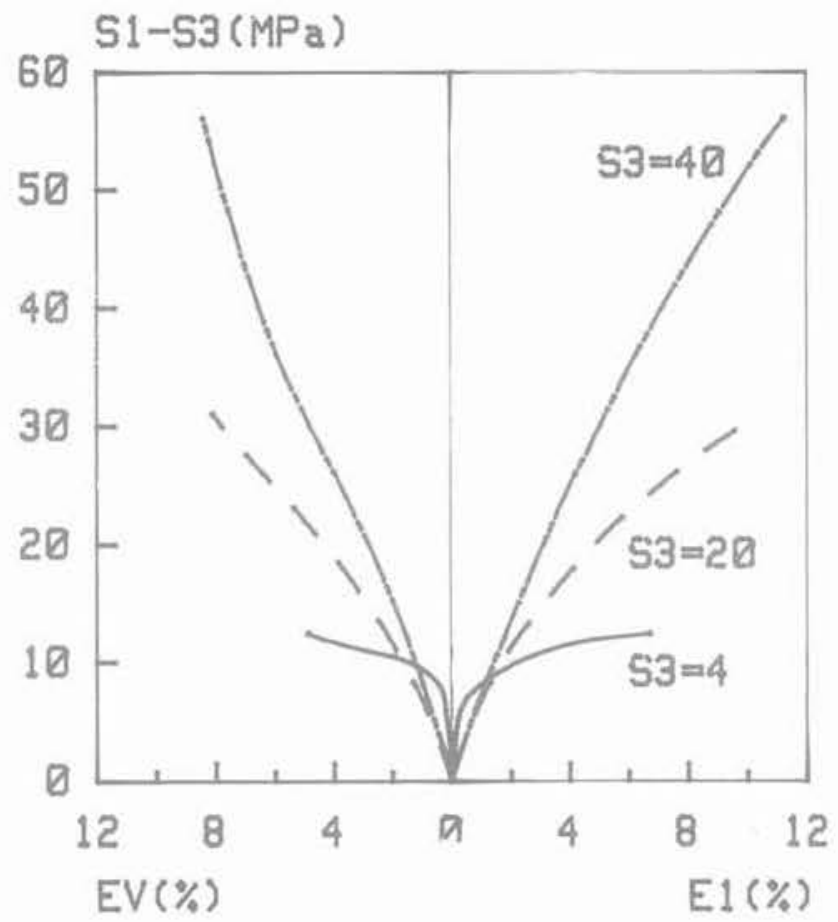

Fig. 3. - Essais triaxiaux en compression.

Fig. 3. - Conventional triaxial compression tests.

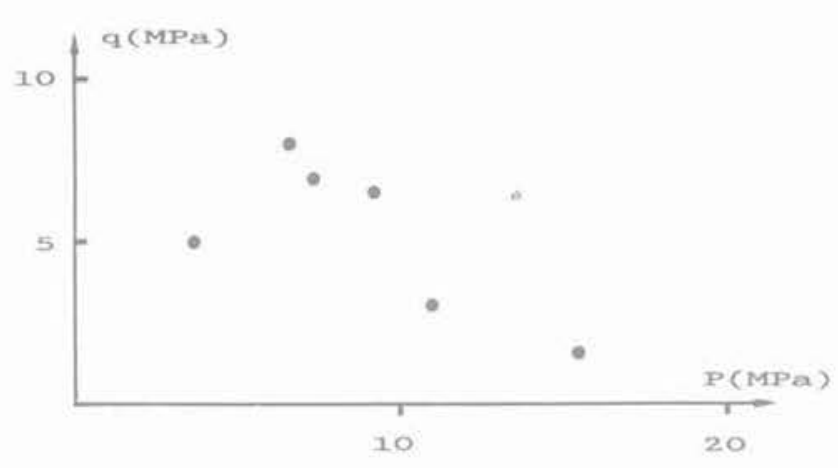

Fig. 4. - Variation de la limite d'élasticité initiale. Fig. 4. - Variation of initial elastic limit.

courbe contraintes-déformations. Par contre, pour les essais à pression de confinement supérieure à $\mathrm{P}_{c}^{\circ}$, ces pics n'existent plus. Dans ces cas, nous avons défini des états ultimes conventionnels par l'apparition d'une déformation axiale permanente importante de l'ordre de $12 \%$.

2. Dans les essais à faibles pressions de confinement $\left(\leqslant \mathrm{P}_{\mathrm{c}}^{\circ}\right)$, il existe une phase élastique initiale plus ou moins importante. Cette limite d'élasticité initiale varie en fonction de la pression de confinement, ce qui est montré dans la figure 4 . Remarquons que cette phase élastique initiale devient négligeable lorsque la pression de confinement dépasse $\mathrm{P}_{\mathrm{c}}^{\circ}$. De tels résultats avaient déjà été obtenus antérieurement [2] [3]

3. Dans tous les essais de compression triaxiale effectués sur la craie, nous avons constaté que, quelle que 
soit la valeur de la pression de confinement appliquée, cette roche poreuse présente toujours une contractance plus ou moins forte.

4. Dans chaque essai triaxial, nous avons effectué en moyenne 10 cycles de déchargement-rechargement partiel. Ceci permet d'évaluer le module d'élasticité $E_{u t}$ dont les valeurs expérimentales sont données par la figure 5. Nous constatons que le module d'élasticité dépend de la pression de confinement d'une part, et d'autre part du déviateur de contraintes.

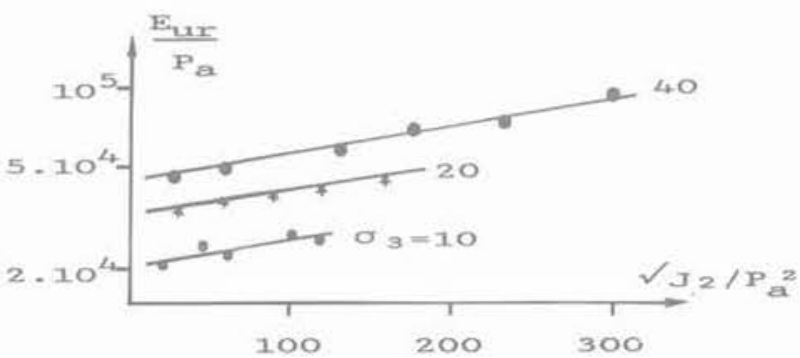

Fig. 5. - Variation du module d'élasticité.

Fig. 5. - Variation of elastic modulus.

\section{ADAPTATION DU MODÈLE ÉLASTOPLASTIQUE DE LADE A LA MODÉLISATION DE LA CRAIE}

L'étude expérimentale effectuée a montré que l'effrondrement plastique progressif de la microstructure et la forte contractance due aux cisaillements font les difficultés majeures de la modélisation du comportement de la craie. LADE a élaboré un modèle élastoplastique à deux surfaces de charge pour modéliser le comportement des sables [5], c'est ce modèle élastoplastique qui a été choisi comme base pour la modélisation du comportement de la craie, et ce pour les raisons suivantes :

1. Le mécanisme de déformation plastique contractante du modèle de LADE peut être, avec quelques modifications nécessaires, très convenable pour décrire la destruction progressive de la microstructure de la craie.

2. Le modèle de LADE est un modèle élastoplastique à écrouissage isotrope donc l'écriture des règles d'écrouissage est très simple. Ceci suffit dans les applications de mécanique des roches où les chargements sont le plus souvent monotones. Ainsi l'écrouissage anisotrope, éventuellement induit par l'histoire du chargement, nécessaire pour décrire le déchargement n'est pas forcément utile.

3. Le critère de rupture introduit dans le mécanisme de déformation plastique déviatorique a déjà été appliqué aux bétons et roches [6]. La cohérence du critère avec de nombreux résultats expérimentaux justifie ce choix.

En tenant compte des particularités du comportement de la craie, l'adaptation du modèle de LADE à la modélisation de cette roche porte essentiellement sur les points suivants :

- prise en considération de la résistance en traction simple de la craie, - prise en compte de la destruction progressive de la microstructure dans la description des règles d'écrouissage,

- simplification du potentiel plastique et de la règle d'écrouissage déviatorique.

D'après le modèle de LADE, la déformation totale est la somme d'une déformation élastique $\varepsilon_{\mathrm{j}}^{\mathrm{e}}$, d'une déformation contractante $\varepsilon_{\text {ii, }}^{\mathrm{c}}$ et d'une déformation plastique déviatorique $\varepsilon_{i j}^{d}$. Sous forme incrémentale, nous avons l'expression suivante :

$$
\mathrm{d} \varepsilon_{i j}=\mathrm{d} \varepsilon_{i j}^{e}+\mathrm{d} \varepsilon_{i j}^{c}+\mathrm{d} \varepsilon_{i j}^{\mathrm{d}}
$$

\subsection{Déformation élastique}

Pour un matériau isotrope homogène, la déformation élastique est calculée avec la loi de HOOKE :

$$
\mathrm{d} \varepsilon_{i j}^{e}=C_{i j k l}^{e} \mathrm{~d} \sigma_{\mathrm{k}}
$$

où $\mathrm{C}_{\text {ik }}^{e}$ est le tenseur d'élasticité qui peut être déterminé par le module d'élasticité $\mathrm{E}_{u}$ et le module de compressibilité isotrope $\mathrm{K}_{u \mathrm{ur}}$. Nous avons déjà évoqué que les modules $\mathrm{E}_{\mathrm{ur}}$ et $\mathrm{K}_{\mathrm{ur}}$ dépendent de la pression de confinement d'une part, et du déviateur de contraintes d'autre part dans le cas de $\mathrm{E}_{\text {ur. }}$. Les relations suivantes ont été proposées pour traduire ces dépendances:

$$
\begin{array}{ll}
E_{u r}=E \cdot\left(\sigma_{3} / P_{c}^{0}\right)^{N_{c}} \cdot 10^{\left(A \sqrt{J_{2} / P_{a^{2}}} / 1000\right)}, & E_{u r} \geq E \\
K_{u r}=K \cdot\left(I_{1} / 3 P_{c}^{0}\right)^{N c} & , K_{u r}>K
\end{array}
$$

où $\mathrm{E}$ et $\mathrm{K}$ sont les valeurs initiales de $\mathrm{E}_{\mathrm{ur}}$ et $\mathrm{K}_{\mathrm{ur}}$ dans le domaine d'élasticité initial ; $A$ et $N_{c}$ deux paramètres du matériau. Les figures 2 et 5 montrent les droites de corrélation qui permettent de déterminer les valeurs des paramètres $\mathrm{N}_{c}$ et $\mathrm{A}, \mathrm{P}_{\mathrm{a}}$ représente la pression atmosphérique exprimée dans la même unité que les contraintes. $I_{1}$ et $J_{2}$ sont le premier et le deuxième invariants du tenseur de contraintes et de son déviateur respectivement.

\subsection{Changement de repère}

Afin de prendre en compte la résistance en traction simple dans la modélisation du comportement de la craie, nous avons procédé à un changement de repère correspondant à une translation des axes principaux de contraintes suivant la direction d'extension de l'axe hydrostatique d'une distance supposée égale à la résistance en traction de cette roche. Nous avons en conséquence :

$$
\overline{\sigma_{i j}}=\sigma_{i j}+C_{0} \cdot P_{a} \cdot \delta_{1 j}
$$

où $\mathrm{C}_{0}, \mathrm{P}_{\mathrm{n}}$ est la résistance en traction de la craie dont la valeur a été déterminée par des essais brésiliens.

\subsection{Déformation plastique contractante}

Le mécanisme de déformation plastique contractante traduit essentiellement l'effondrement plastique des pores et la forte contractance irréversible de la craie. C'est le terme prépondérant dans l'ensemble de trois composantes de déformation de la craie lorsque la pression hydrostatique est importante $\left(>\mathrm{P}_{\mathrm{c}}^{\circ}\right)$. La déformation plastique contractante est déterminée par une loi d'écoulement associée et une règle d'écrouissage isotrope. 
Dans le repère transformé, la fonction de charge contractante est exprimée par la relation suivante :

$$
F_{c}=T_{1}^{2}+2 \bar{I}_{2}-Y_{c}=0
$$

où $\overline{\mathrm{I}}_{1}$ et $\overline{\mathrm{I}}_{2}$ sont le premier et le deuxième invariant du tenseur de contraintes déterminé dans le nouveau repère. $Y_{c}$ est une fonction croissante et monotone représentant l'état actuel d'écrouissage du matériau.

La règle d'écrouissage isotrope contractant est représentée par la relation suivante :

$$
\mathrm{Y}_{\mathrm{c}}=\mathrm{Y}_{\mathrm{c}}^{\circ}+\mathrm{C} \cdot \mathrm{P}_{\mathrm{a}}^{2} \cdot\left(\mathrm{W}_{\mathrm{c}} / \mathrm{P}_{\mathrm{a}}\right)_{\rho}
$$

Dans cette relation, $Y_{c}{ }^{\circ}$ est la valeur initiale de $Y_{c}$, correspondant à la limite élastique en compression isotrope $\mathrm{P}_{c}, \mathrm{~W}_{\mathrm{c}}$ est l'énergie de déformation plastique contractante, déterminée à l'aide des cycles de déchargement-rechargement effectués dans un essai de compression isotrope, $C$ et $\rho$ sont deux paramètres sans dimension du matériau. Dans la figure 6 , nous avons présenté les valeurs de $W_{c}$ en fonction de $Y_{c}$ ainsi que la courbe de corrélation qui permet de déterminer les valeurs des paramètres $C$ et $\rho$. Afin de prendre en considération la destruction progressive de microstructure de la craie, nous avons pris deux valeurs différentes, respectivement pour $C$ et $\rho$, dans les deux zones d'écrouissage distinctes délimitées par $Y_{c}^{\prime}$ correspondant à la limite d'effrondrement plastique $P_{c}^{i}$. Nous avons donc :

$$
\begin{aligned}
& \mathrm{C}=\mathrm{C}_{1} \cdot \rho=\rho_{1} . \mathrm{Y}_{\mathrm{c}} \overline{\bar{Z}} \mathrm{Y}_{\mathrm{c}}^{\mathrm{t}} \\
& \mathrm{C}=\mathrm{C}_{2} \cdot \rho=\rho_{2} . \mathrm{Y}_{\mathrm{c}}>\mathrm{Y}_{\mathrm{c}}^{\mathrm{t}}
\end{aligned}
$$

avec $\quad Y_{c}^{t}=3\left(P_{c}^{t}+C_{0} \cdot P_{a}\right)^{2}$

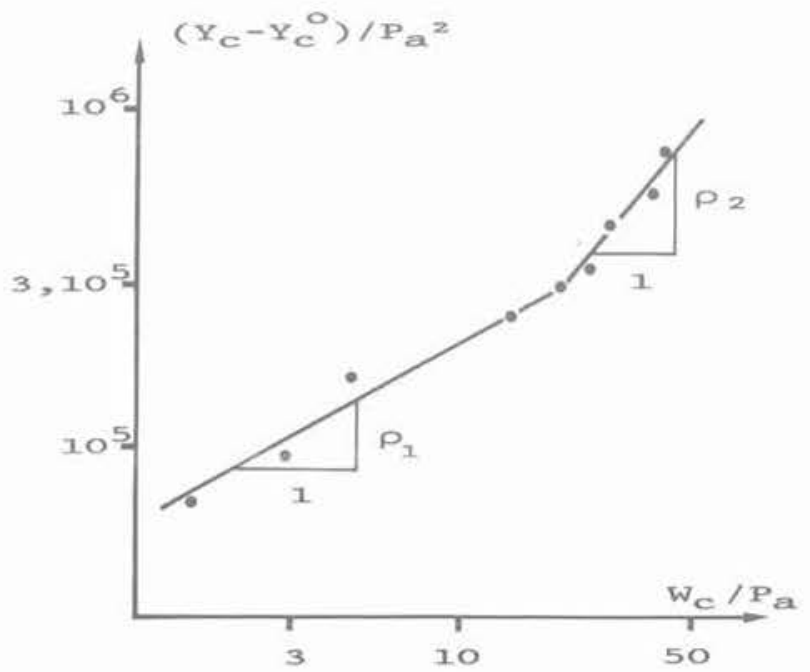

Fig. 6. - Variation de $Y_{c}$ en fonction de $W_{c}$ et corrélation. Fig. 6. - Variation of $Y_{c}$ with $W_{c}$ and correlation.
La condition de continuité de la fonction (7) entre deux zones d'écrouissage est assurée par :

$$
C_{2}=\left(Y_{c}^{t}-Y_{c}^{\circ}\right) / P_{a}^{2} \cdot\left[\left(Y_{c}^{i}-Y_{c}^{0}\right) /\left(C_{1} \cdot P_{a}^{2}\right)\right]-\left(c_{2} / \rho_{1}\right)
$$

L'utilisation d'une loi d'écoulement contractant associée implique que le potentiel plastique contractant est identique à la fonction de charge contractante, soit :

$$
\mathrm{Q}_{\mathrm{c}}=\overline{\mathrm{I}}_{1}^{2}+\overline{2 \mathrm{I}}_{2}
$$

La théorie de plasticité classique permet alors d'exprimer la déformation plastique contractante par :

$$
\mathrm{d} \varepsilon_{\mathrm{i}}=\mathrm{d} \lambda_{\mathrm{c}} \frac{\partial \mathrm{Q}_{\mathrm{c}}}{\partial \sigma_{\mathrm{l}}}, \mathrm{F}_{\mathrm{c}}=0 \text { et } \frac{\partial \mathrm{F}_{\mathrm{c}}}{\partial \sigma} \mathrm{d} \sigma>0
$$

Le multiplicateur $\mathrm{d} \lambda_{c}$ est déterminé par la condition de consistance, $\mathrm{dF}_{\mathrm{c}}=0,[10]$.

\subsection{Déformation plastique déviatorique}

La déformation plastique déviatorique est due aux cisaillements des ponts entre les grains. Elle est déterminée par une loi d'écoulement non associée et une règle d'écrouissage isotrope. Ce mécanisme de déformation plastique contrôle entièrement la rupture du matériau. Nous avons utilisé le critère de rupture de LADE, proposé pour les roches et les bétons. Dans le nouveau repère celui-ci s'écrit :

$$
\left(\overline{I_{1}} 3 / \overline{T_{3}}-27\right)\left(\overline{I_{1}} / P_{\mathrm{a}}\right)^{m}-Y_{d}^{r}=0
$$

où $\mathrm{I}_{3}$ est le troisième invariant du tenseur de contraintes, $m$ et $Y_{d}$ sont deux paramètres de rupture du matériau. Dans la figure 7, nous avons montré la relation expérimentale entre $\left(\overline{\mathrm{I}_{1}} 3 / \overline{\mathrm{I}_{3}}-27\right)$ et $\left(\mathrm{P}_{\mathrm{a}} / \overline{\mathrm{I}_{1}}\right)$ pour les contraintes à la rupture obtenues dans la série d'essais triaxiaux, ainsi que la courbe de corrélation qui permet de déterminer les valeurs des paramètres $m$ et $\mathrm{Y}_{\mathrm{d}}^{\mathrm{r}}$. La figure 8 montre la comparaison entre la courbe de rupture donnée par le critère (13) et les valeurs expérimentales des contraintes à la rupture, dans le plan des essais triaxiaux de révolution. Nous constatons une bonne concordance entre ces deux résultats.

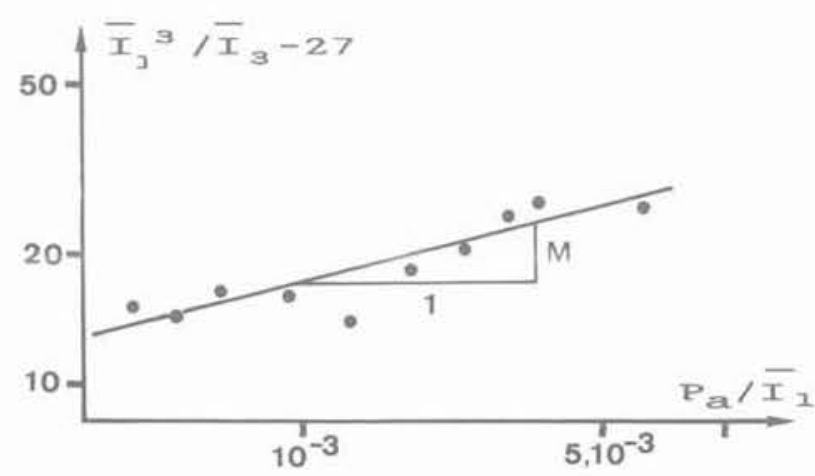

Fig. 7. - Détermination du critére de rupture. Fig. 7. - Determination of failure criterion. 


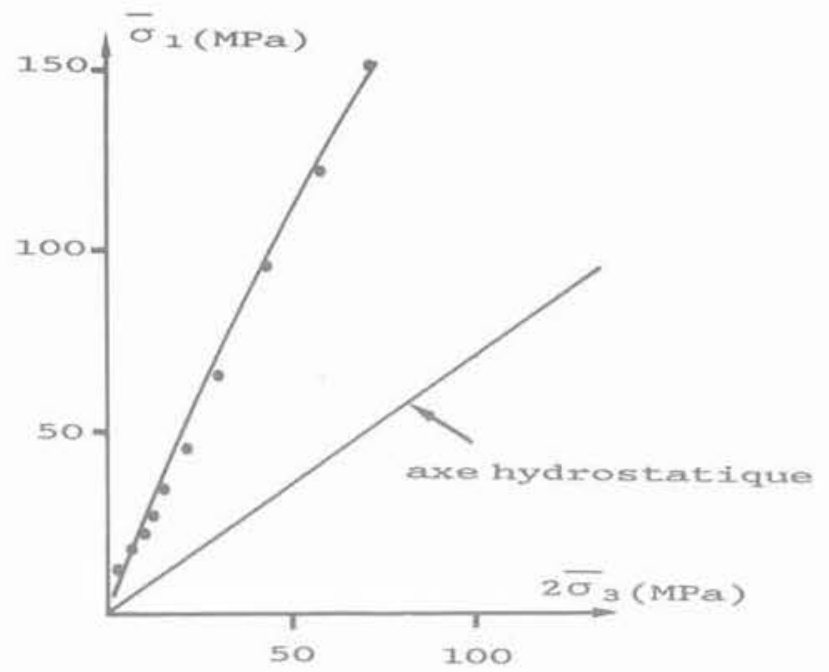

Fig. 8. - Courbe de rupture dans le plan des essais triaxiaux et verification.

Fig. 8. - Failure curve in triaxial plane and verification.

Avec l'hypothèse que la surface de rupture est la position ultime de la surface de charge, nous pouvons écrire la fonction de charge déviatorique par l'expression suivante :

$$
\left(\overline{I_{1}} 3 / \overline{I_{3}}-27\right)\left(\overline{I_{1}} / P_{a}\right)^{m}-Y_{d}=0
$$

où $Y_{d}$ est une fonction monotone croissante de l'état d'écrouissage du matériau, dont la valeur ultime est celle de $Y_{\mathrm{d}}^{r}$.

La règle d'écrouissage déviatorique est définie par la

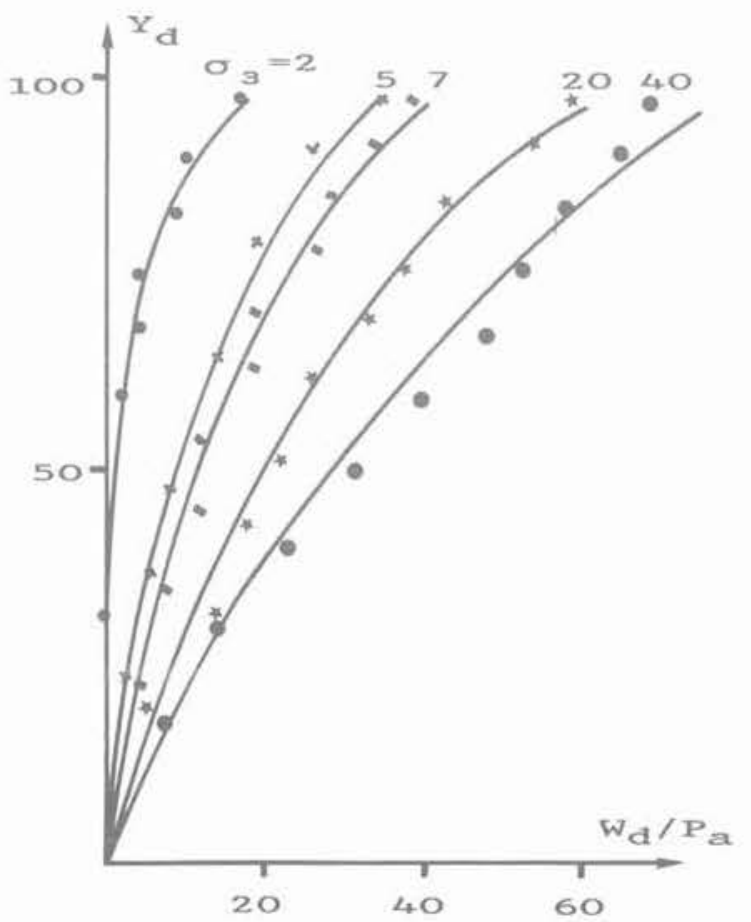

relation suivante :

$$
Y_{d}=Y_{d}^{o}+\left(Y_{d}^{r}-Y_{d}^{\circ}\right)\left(W_{d} / W_{d}^{r}\right)^{q}
$$

où $\mathrm{Y}_{\mathrm{d}}^{\circ}, \mathrm{W}_{\mathrm{d}}^{\mathrm{t}}, \mathrm{q}$ sont trois constantes dans un essai triaxial de révolution. $W_{f}$ est l'énergie de déformation plastique déviatorique dont les valeurs sont déterminées à l'aide des cycles de déchargement-rechargement effectués dans les essais triaxiaux. La figure 9 montre les valeurs expérimentales de $W_{d}$ en fonction de $Y_{\mathrm{d}}$ et la courbe de corrélation pour chaque pression de confinement.

$Y_{\mathrm{d}}^{\circ}$ représente la limite d'élasticité initiale du matériau. Ses valeurs sont obtenues par intersection de laxe $Y_{\text {d }}$ et des courbes de corrélation de la figure $9 . W_{d}{ }^{\top}$, l'énergie de déformation plastique déviatorique à la rupture est déterminée de façon semblable. Le taux d'écrouissage q est calculé, pour une pression de confinement donnée, par l'équation suivante :

$$
q=\log \left[\left(Y_{d}^{r}-Y_{d}^{\circ}\right) /\left(Y_{d}^{60}-Y_{d}^{\circ}\right)\right] / \log \left(W_{d}^{r} / W_{d}^{60}\right)
$$

où

$$
Y_{d}^{60}=Y_{d}^{o}+0,6\left(Y_{d}-Y_{d}^{o}\right)
$$

Il est évident que $\mathrm{Y}_{\mathrm{d}}{ }^{\circ}, \mathrm{W}_{\mathrm{d}}$ et $\mathrm{q}$ dépendent de la pression de confinement. Nous proposons alors de décrire ces variations sous la forme :

$$
\begin{gathered}
\mathrm{Y}_{\mathrm{d}}=\lambda\left(\sigma_{3}-\mathrm{P}_{\mathrm{c}}^{\circ}\right) / \mathrm{P}_{\mathrm{a}} \cdot \mathrm{Y}_{\mathrm{d}} \geqq 0 \\
\mathrm{~W}_{\mathrm{d}}^{\mathrm{s}}=\mathrm{P}_{1} \cdot \mathrm{P}_{\mathrm{a}} \cdot\left[\left(\sigma_{3}+\mathrm{P}_{\mathrm{a}}\right) / \mathrm{P}_{\mathrm{a}}\right]^{1} \\
\mathrm{q}=\alpha \cdot\left[\left(\sigma_{3}+\mathrm{P}_{\mathrm{a}}\right) / \mathrm{P}_{\mathrm{a}}\right]^{\beta}
\end{gathered}
$$

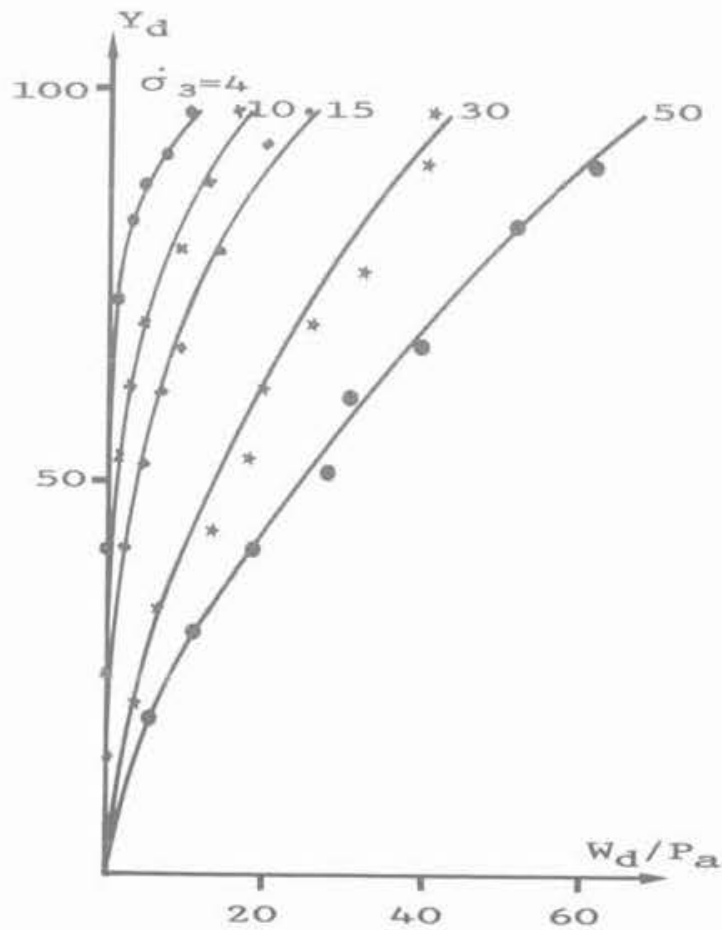

Fig. 9. - Variation de $Y_{d}$ en fonction de $W_{d}$.

Fig. 9. - Variation of $Y_{d}$ with $W_{d}$ 
Dans les figures $10,11,12$, nous présentons respectivement les valeurs de $\mathrm{Y}_{d}{ }^{\circ}, \mathrm{W}_{\mathrm{d}}{ }^{\mathrm{t}}$ et de $\mathrm{q}$, calculées à partir des courbes de la figure 9 , en fonction de la pression de confinement. Les valeurs des paramètres du maté. riau $\lambda, P_{1}, 1, \alpha$ et $\beta$ sont ensuite déterminées par les droites de corrélation des figures 10,11 et 12 .

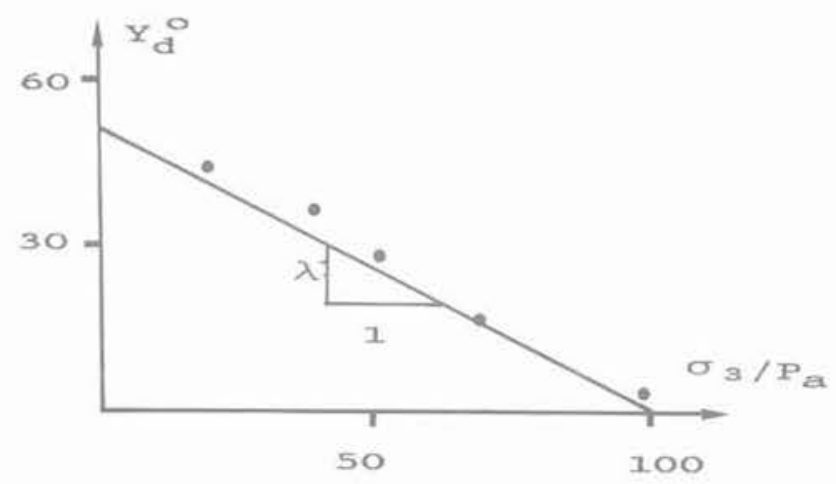

Fig. 10. - Variation de $\gamma_{d}{ }^{0}$ en fonction de $\sigma_{3}$ Fig. 10. - Variation of $Y_{d}^{o}$ with $\sigma_{3}$

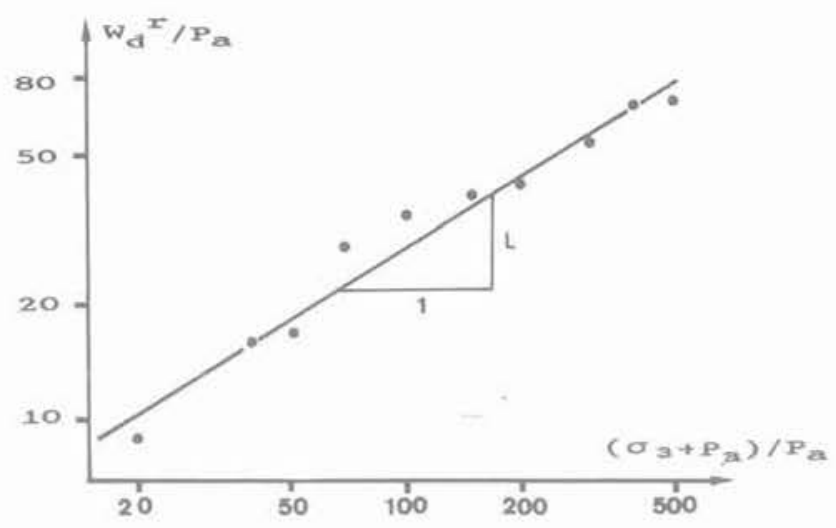

Fig. 11. - Variation de $W_{d}^{\prime}$ en fonction de $\sigma_{3}$. Fig. 11. - Variation of $W_{d}^{\prime}$ with $\sigma_{3}$

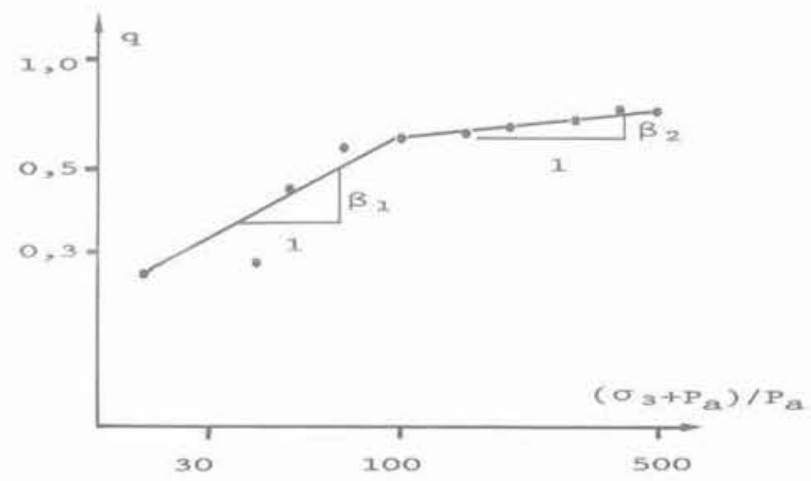

Fig. 12. - Variation de $q$ en fonction de $\sigma_{3}$ Fig. 12. - Variation of $q$ with $\sigma_{3}$.
Nous avons déjà évoqué que le comportement de la craie dans des essais à faibles pressions de confinement $\left(<\mathrm{P}_{\mathrm{c}}^{\circ}\right)$ est très différent de celui dans des essais à des pressions de confinement élevées $\left(>\mathrm{P}_{\mathrm{c}}^{\circ}\right)$. Pour restituer cette différence, nous avons proposé respectivement deux valeurs différentes pour $\alpha$ et $\beta$ pour les deux zones d'écrouissage délimitées par $\mathrm{P}_{c}^{\circ}$, soit :

$$
\begin{aligned}
& \alpha=\alpha_{1} \cdot \beta=\beta_{1} \cdot \sigma_{3} \leqq \mathrm{P}_{\mathrm{c}}^{\circ} \\
& \alpha=\alpha_{2} \cdot \beta=\beta_{2} \cdot \sigma_{3}>\mathrm{P}_{\mathrm{c}}^{\circ}
\end{aligned}
$$

avec :

$$
\alpha_{2}=\alpha_{1}\left(\frac{\mathrm{P}_{c}^{\circ}+\mathrm{P}_{\mathrm{a}}}{\mathrm{P}_{\mathrm{a}}}\right)^{\left(\beta_{1}-\beta_{2}\right)}
$$

Après avoir effectué une étude d'influence des paramètres du potentiel plastique de LADE [5] sur la modélisation du comportement de la craie, nous proposons une simplification de celui-ci [10] sous la forme :

$$
Q_{d}=\bar{I}_{1}^{3}-27 \overline{\mathrm{I}_{3}}
$$

La déformation plastique déviatorique se calcule alors par :

$$
\mathrm{d} \varepsilon_{\mathrm{i}}^{\mathrm{d}}=\mathrm{d} \lambda_{\mathrm{d}} \frac{\partial \mathrm{Q}_{\mathrm{d}}}{\partial \sigma_{i j}} \cdot \mathrm{F}_{\mathrm{d}}=0 \text { et } \frac{\partial \mathrm{F}_{\mathrm{d}}}{\partial \sigma} \mathrm{d} \sigma>0
$$

Le multiplicateur $\mathrm{d} \lambda_{\mathrm{d}}$ est déterminé par la condition de consistance, $\mathrm{dF}_{\mathrm{d}}=0$, [10].

\section{Tableau 1. - Récapitulation des paramètres du modèle pour la craie étudiée. \\ Table 1. - Summary of material constants for studied porous chalk.}

\begin{tabular}{|l|l|l|}
\hline $\begin{array}{c}\text { paramètres } \\
\text { d'élasticité }\end{array}$ & \multicolumn{1}{c|}{$\begin{array}{c}\text { plasticité } \\
\text { contractante }\end{array}$} & \multicolumn{1}{c|}{$\begin{array}{c}\text { plasticité } \\
\text { déviatorique }\end{array}$} \\
\hline $\mathrm{E}=2000 \mathrm{MPa}$ & $\mathrm{C}_{1}=55528$ & $\mathrm{~m}=0,25$ \\
$\mathrm{~K}=1400 \mathrm{MPa}$ & $P_{1}=0,565$ & $\mathrm{Y}_{\mathrm{d}}^{\prime}=97,43$ \\
$\mathrm{C}_{0}=7,0$ & $P_{2}=1,22$ & $\lambda=-0,51$ \\
$\mathrm{~A}=1,01$ & $\mathrm{P}_{\mathrm{c}}^{\mathrm{t}}=32,5 \mathrm{MPa}$ & $\mathrm{P}_{1}=1,60$ \\
$\mathrm{~N}_{\mathrm{c}}=0,54$ & $\mathrm{P}_{\mathrm{c}}^{0}=10 \mathrm{MPa}$ & $1=0,624$ \\
& & $\alpha_{1}=0,044$ \\
& & $\beta_{1}=0,57$ \\
& & $\beta_{2}=0,11$ \\
\hline
\end{tabular}


3.5. Simulation des essais de compression isotrope et triaxiale à l'aide du modèle

Tous les paramètres du modèle ont été déterminés à partir des essais de compression isotrope et triaxiale de révolution. Pour la craie étudiée, les valeurs des paramètres sont résumées dans le tableau 1. Ces essais ont ensuite été simulés à l'aide du modèle de LADE modifié et avec les valeurs des paramètres ainsi obtenues [10]. Cette simulation permet ou non de justifier la bonne qualité de la détermination des paramètres pour décrire les aspects fondamentaux du comportement de la craie. Dans les figures 13 et 14 , nous avons présenté la comparaison entre l'expérience et la simulation pour un essai de compression isotrope et les trois essais de compression triaxiale représentatifs. Nous constatons une très bonne concordance entre les deux résultats.

\section{MODÉLISATION DU COMPORTEMENT DE LA CRAIE DANS DES PROBLĖMES HOMOGENNES}

Deux types d'essais ont été effectués pour étudier le comportement de la craie dans des problèmes homogènes. Ce sont des essais de sollicitations proportionnelles et l'essai triaxial d'extension latérale. Ces essais ont ensuite été simulés à l'aide du modèle de LADE modifié.

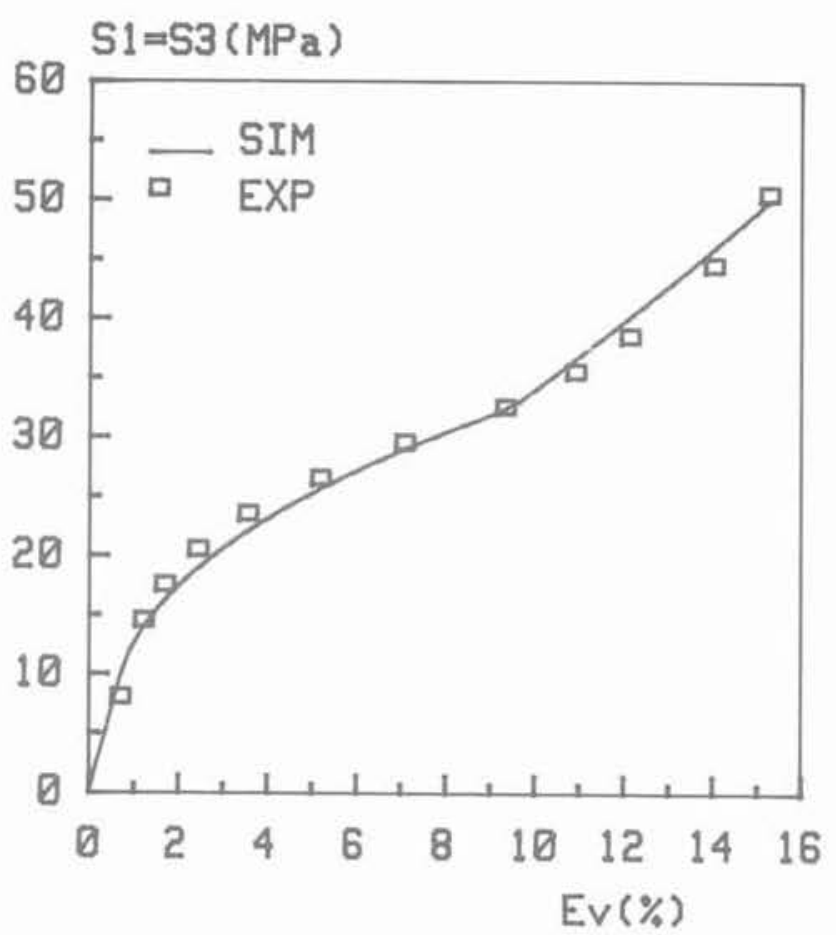

Fig. 13. - Simulation d'un essai de compression isotrope. Fig. 13. - Simulation of hydrostatic compression test.
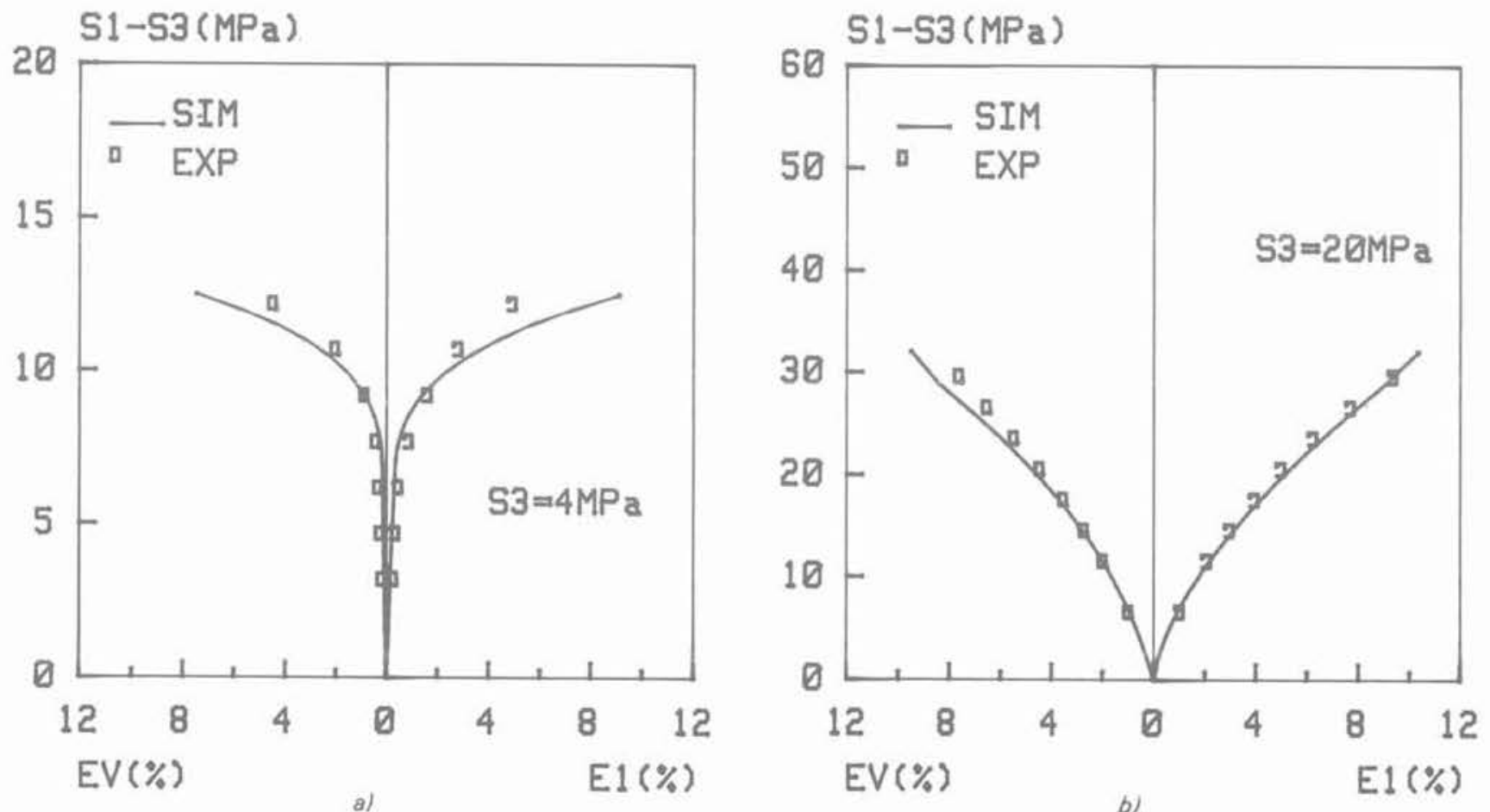

Fig. 14. - Simulation des essais triaxiaux.

Fig. 14. - Simulation of triaxial compression tests. 

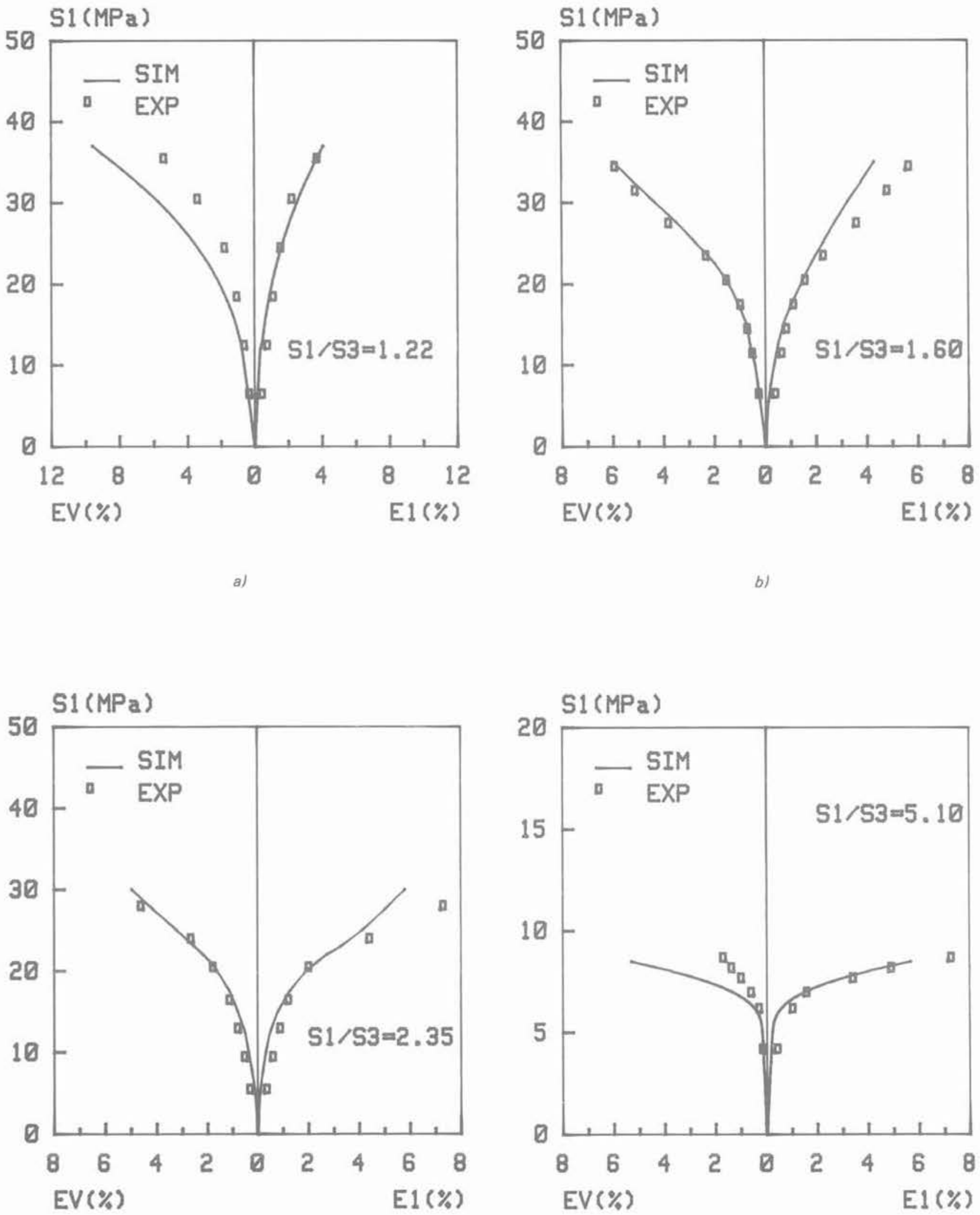


\section{$\mathrm{S} 1-\mathrm{S3}(\mathrm{MPa})$}

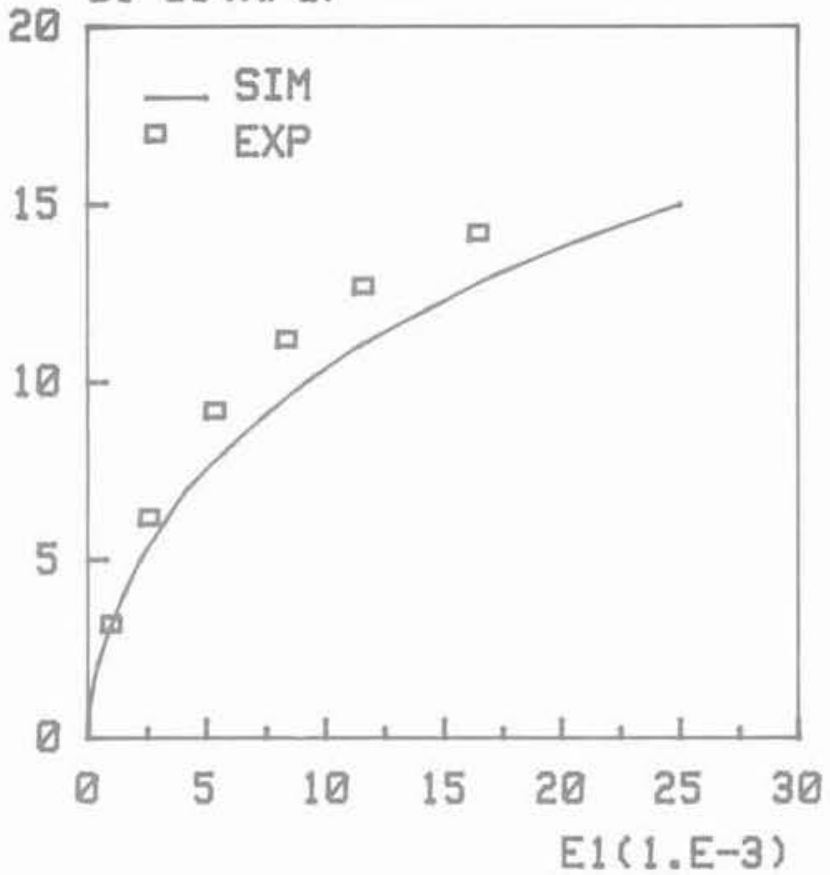

\section{$51-53(\mathrm{MPa})$}

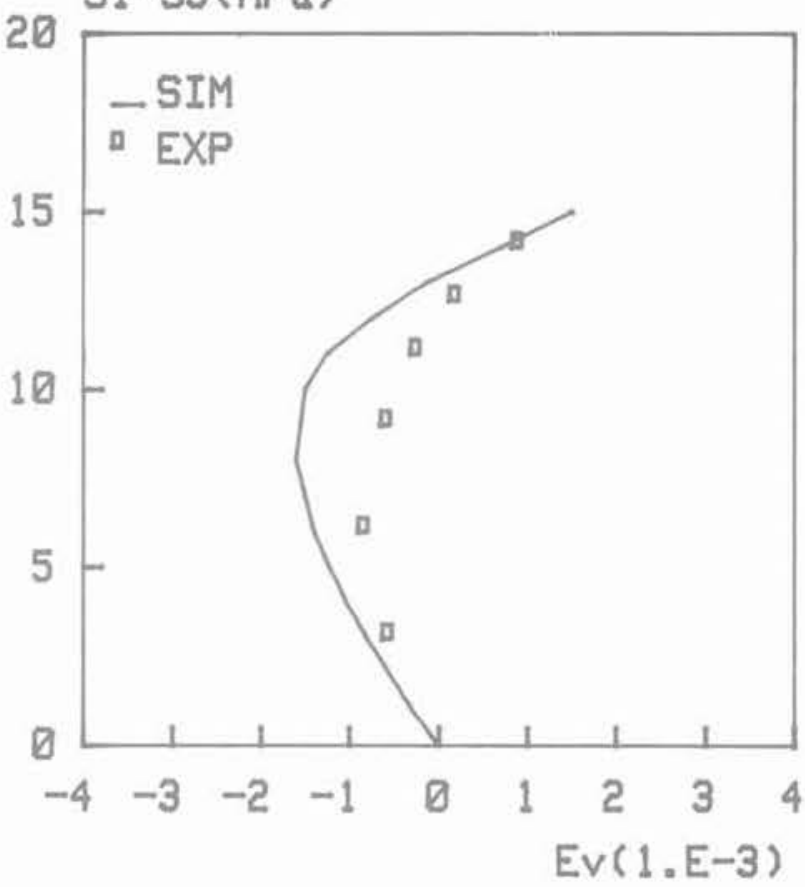

b)

Fig. 16. - Simulation d'un essai triaxial d'extension latérale

(a) déformation axiale,

(b) déformation volumique.

Fig, 16, - Simulation of reduced triaxial compression test

(a) axial strain.

(b) volumetric strain.

\subsection{Essais de sollicitations proportionnelles}

Il consiste à effectuer un essai triaxial de révolution particulier dans lequel on fait varier simultanément les contraintes axiale et latérale avec un rapport fixe $k\left(k=\sigma_{1} / \sigma_{3}\right)$. Dans la figure 15 , nous avons présenté les résultats expérimentaux par les courbes de contraintes - déformations axiales $\left(\sigma_{1}-\varepsilon_{1}\right)$ et de contraintesdéformations volumiques $\left(\sigma_{1}-\varepsilon_{y}\right)$. Les résultats obtenus par la simulation de ces essais à l'aide du modèle élastoplastique sont présentés dans la même figure pour différentes valeurs du rapport $k$. D'après ces comparaisons, nous remarquons une bonne concordance entre l'expérience et la simulation numérique tant pour la déformation axiale que pour la déformation volumique. Néanmoins, il est constaté que la précision de la simulation est d'autant meilleure que le rapport $k$ est faible.

\subsection{Essai triaxial d'extension latérale}

Il s'agit d'un essai triaxial au cours duquel, à partir d'un état isotrope, on diminue, incrémentalement la pression de confinement en gardant la force axiale constante. Le modèle de LADE modifié a été utilisé pour simuler cet essai. Dans la figure 16 , nous avons présenté les déformations axiale et volumique en fonction du déviateur de contrainte obtenues par l'expérience et les résultats de la simulation. Nous constatons une très bonne concordance entre les deux résultats pour la relation entre le déviateur de contraintes et la déformation axiale. En ce qui concerne le comportement dévia- teur de contraintes - déformation volumique, nous avons également une cohérence satisfaisante bien qu'il existe un certain écart entre l'expérience et la simulation dans la phase de dilatance.

\section{MODÉLISATION DU COMPORTEMENT DE LA CRAIE DANS DES PROBLĖMES AUX LIMITES TYPES}

Les essais sur cylindres creux ont été choisis pour étudier le comportement de la craie dans des problèmes aux limites types. Les cotes géométriques des cylindres creux testés étaient les suivants :

diamètre extérieur: $110 \mathrm{~mm}$, diamètre intérieur : $40 \mathrm{~mm}$, hauteur : $120 \mathrm{~mm}$.

Les éprouvettes ont été très soigneusement usinées. Un système de mise en place des éprouvettes cylindriques dans la cellule a spécialement été conçu [10] qui permet de faire varier indépendamment la pression extérieure $\left(\mathrm{P}_{e}\right)$, la pression intérieure $\left(\mathrm{P}_{1}\right)$ et la force axiale $\left(\mathrm{F}_{\mathrm{s}}\right)$.

Afin de simuler ces essais avec le modèle de LADE modifié, ce dernier a été introduit dans un programme de calcul par éléments finis [10].

Deux types d'essais sur cylindres creux ont été effectués ; l'essai en pression extérieure contrôlée et l'essai en force axiale contrôlée. 


\subsection{Essai en pression extérieure contrôlée}

Dans cet essai, le cylindre est d'abord soumis à un état de contrainte isotrope dans la zone élastique $\left(P_{e}=P_{1}<P_{c}^{\circ}\right)$. On augmente ensuite les pressions extérieure et intérieure alternativement. Le piston de la presse n'est pas en contact avec l'embase supérieure du cylindre creux. La figure 17 donne la variation de volume en fonction de la force appliquée sur la face supérieure du cylindre creux. Dans la même figure sont présentés les résultats de la simulation numérique par éléments finis. Le modèle permet encore de prédire correctement le comportement de la craie.

\subsection{Essai en force axiale contrôlée}

A partir d'un essai isotrope, on accroît la pression extérieure jusqu'à une certaine valeur. On applique ensuite la force axiale par la presse asservie, en gardant les deux pressions constantes. La figure 18 présente le déplacement axial et la variation de volume en fonction de la force appliquée sur la face supérieure du cylindre creux, obtenus par l'expérience et la simulation à l'aide du programme en éléments finis. La comparaison entre les deux résultats nous permet de constater que le modèle proposé rend très bien compte de la relation entre la variation de volume et la force appliquée. Cependant, il existe un écart assez important entre l'expérience et la simulation pour la relation entre le déplacement axial et la force appliquée.

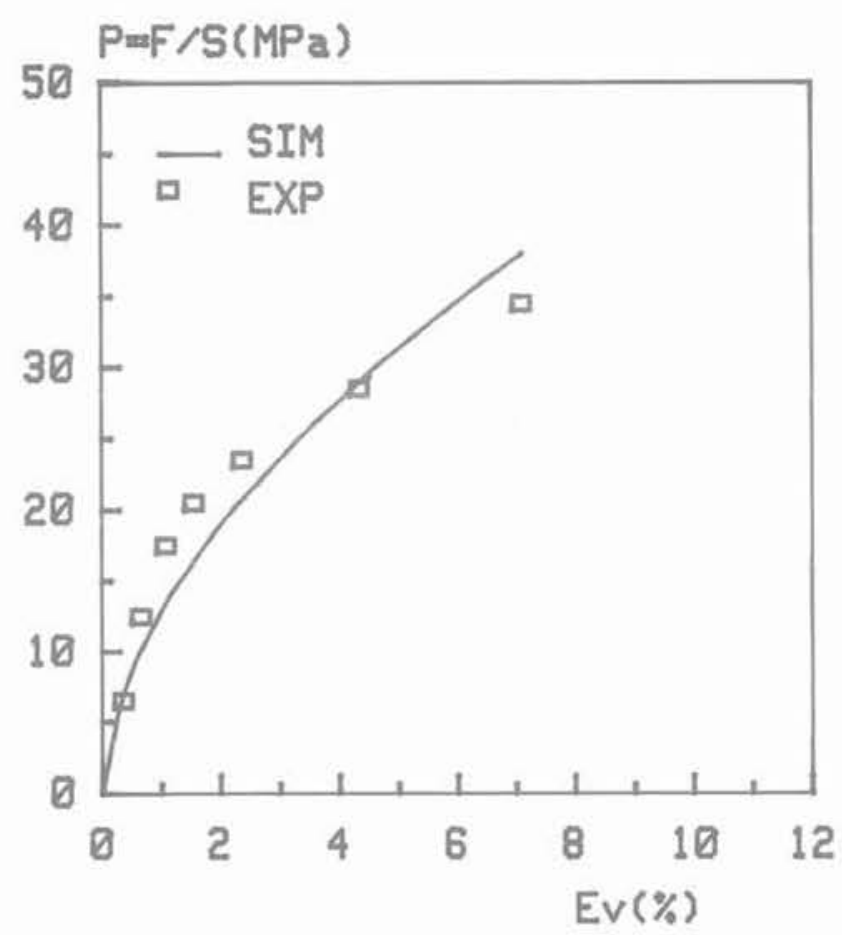

a)

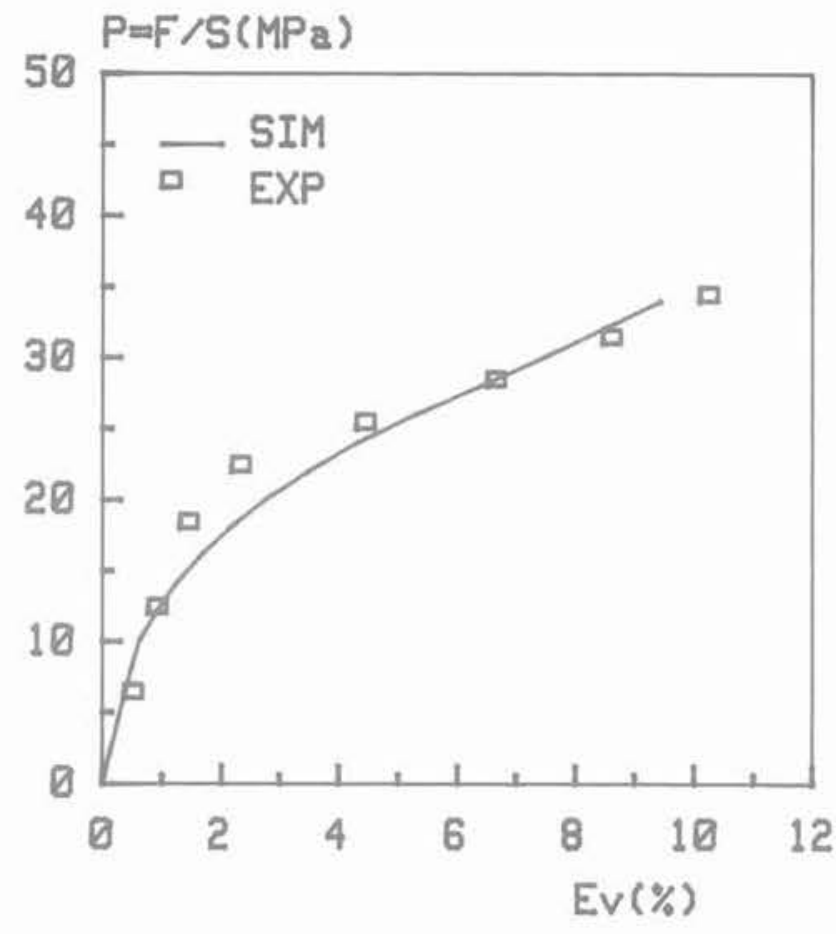

Fig. 17. - Simulation d'un essai sur cylindre creux à pression extérieure controlée.

Fig. 17. - Simulation of hollow cylinder test with exterior pressure control.

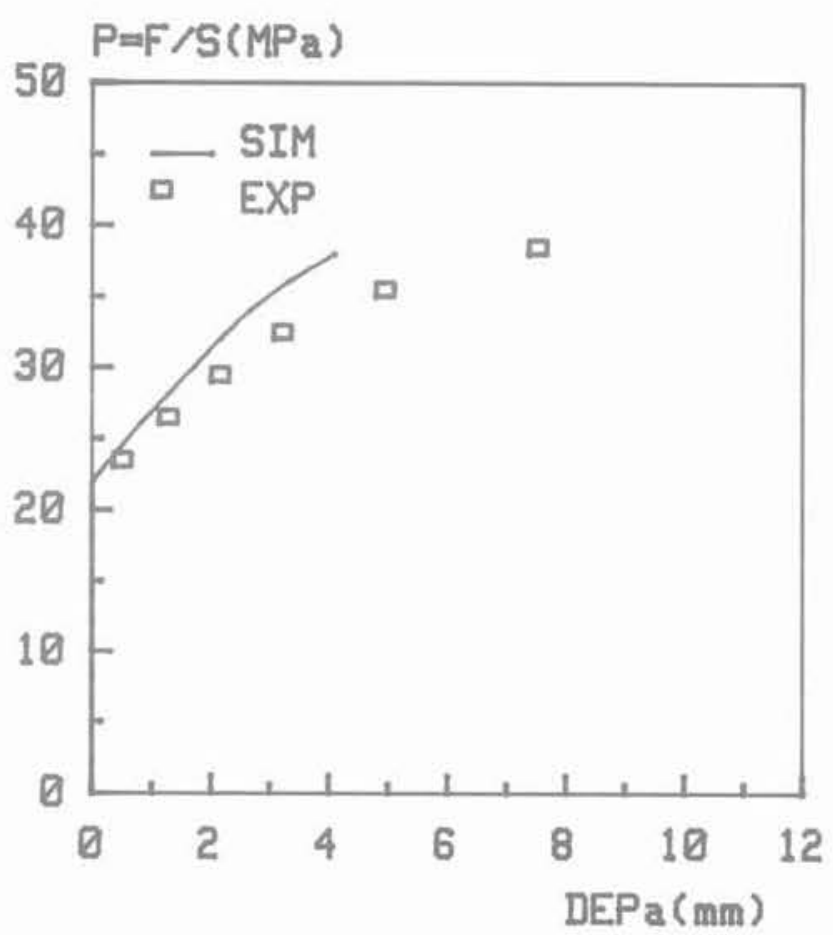

b)

Fig. 18. - Simulation d'un essai sur cylindre creux à force axiale controlée

(a) deformation volumique,

(b) déplacement axial.

Fig. 18. - Simulation of hollow cylinder test with axial loading control.

(a) volumetric strain.

(b) axial displacement. 


\section{CONCLUSION}

Des essais isotropes et triaxiaux en compression effectués sur la craie blanche montrent que le comportement de cette roche poreuse dépend fortement de la pression hydrostatique. Elle présente un comportement intermédiaire entre une roche et un sol, avec une destruction progressive de la structure microporeuse.

Le modèle élastoplastique à deux surfaces de LADE est adapté pour modéliser le comportement de la craie. Tous les paramètres introduits dans le modèle sont déterminés à partir des essais simples : un essai de compression isotrope et des essais triaxiaux en compression. Les modifications apportées au modèle sont justifiées à partir des résultats expérimentaux. Le modèle modifié est introduit dans un programme de calcul par éléments finis.

Des essais de sollicitations proportionnelles et d'extension latérale sont effectués pour tester la validité du modèle proposé pour des problèmes homogènes, et des essais sur cylindres creux pour des champs de contraintes non uniformes. Les bonnes concordances globales entre l'expérience et la simulation numérique permettent de montrer que le modèle proposé prédit correctement le comportement de la craie sous ces types de sollicitations. Néanmoins, d'autres essais de validation du modèle restent à effectuer.

L'intérêt essentiel de la présente modélisation est sa capacité à pouvoir prédire le comportement de la craie dans une gamme de sollicitations. Ceci est nécessaire pour résoudre des problèmes pétroliers à grandes profondeurs (forages profonds et subsidences induites). Mais il est constaté que le nombre de paramètres utilisés dans le modèle est assez élevé. Des simplifications de la formulation du modèle seront donc nécessaires pour ses applications industrielles. En outre, l'introduction des lois d'écrouissage anisotropes et de la viscosité dans le modèle pour prédire le comportement de la craie sous chargements cycliques devra faire l'objet de recherches ultérieures. Enfin, l'utilisation de la plus petite contrainte principale, $\sigma_{3}$, dans la formulation du modèle nécessite aussi des améliorations ultérieures [11].

\section{REMERCIEMENTS}

Les auteurs remercient la Société Nationale Elf Aquitaine pour l'aide apportée à cette étude, et tout particulièrement MM. MAURY et GUENOT pour l'attention et les nombreuses discussions qui ont permis que ce travail aboutisse.

\section{BIBLIOGRAPHIE}

1. CHEATHAM J.P. Jr., ALLEN M.B., CELLE C.C. (1984), Kinematic hardening of a Porous limestone, Rocks mechanics and Rock Engineering, vol. 17, pp. 233-242.

2. ELLIOTT G.M., BROWN E.T. (1985), Yield of $a$ soft high porosity rock, Géotechnique $35, n^{\circ} 4$, pp, 413-423.

3. ELLIOTT G.M., BROWN E.T. (1986), Further development of a plasticity Approach to yield in Porous Rock, I.J.R.M.M.S., vol. 23, n 2 , pp. 151-156.

4. GEROGIANNOPOULOS N.G., BROWN E.T. (1978), The critical state concept applied to rock, I.J.R.M.M.S., vol. 17, pp. 225-229.

5. LADE P.V. (1977), Elastoplastic stress-strain theory for cohesionless soil with curved yield surfaces, J. Eng. Mech. Div. A.S.C.E., vol. 104 $n^{\circ}$ EM5, pp. 850-863.

6. LADE P.V. (1984), Modelling rock strength in three dimension, Int. J. Rock Mech. Min. Sci. \& Geomech. Abstr., vol. 21, $n^{\circ} 1$, pp. 21-33.

7. MILLER T.W., CHEATHAM J.B. Jr. (1972), A new yield condition and hardening rule for rocks, Int. J. Rock. Mech. Min. Sci., vol. 9, pp. $453-474$.

8. MAURY V. (1987), Geomechanical approach of oil and gas production. Main problems, first results, 4th international congress of rock mechanics, Montréal, Canada, September.

9. SHAO J.F., HENRY J.P. (1987), Behaviour and modelisation of porous chalk under confining pressure of up to $50 \mathrm{MPa}$, Second international conference and short course on constitutive laws for engineering materials, janvier, Tucson, U.S.A., pp. $457-465$.

10. SHAO J.F. (1987), Etude du comportement d'une craie blanche très poreuse et modélisation, Thèse de Doctorat, Lille I, 15 septembre 1987.

11. SHAO J.F,, HENRY J.P., GUENOT A. (1988), An adapted consitutive model for soft porous rocks (chalk), proposé pour 29th U.S. Symposium of Rock Mechanics. 